\title{
Discovery of multi-component portfolio strategies with continuous tuning to the changing market micro-regimes using input-dependent boosting
}

\author{
V. V. Gavrishchaka ${ }^{1}$, O. V. Barinova ${ }^{2}$, A. P. Vezhnevets ${ }^{2}$ \\ \& M. A. Monina ${ }^{3}$ \\ ${ }^{1}$ Alexandra Investment Management, New York, NY, USA \\ ${ }^{2}$ Moscow State University, Dept. of Computational Mathematics \\ and Cybernetics, Graphics and Media Lab, Moscow, \\ Russian Federation \\ ${ }^{3}$ Moscow State University of Instrument Engineering and Computer \\ Science, Moscow, Russian Federation
}

\begin{abstract}
Recently proposed boosting-based optimization offers a generic framework for the discovery of compact and interpretable portfolios of complimentary trading strategies with stable (non-resonant) performance over a wide range of market regimes and robust generalization abilities. Inherent complexity control allows the framework to work with very large pools of heterogeneous base strategies with well-established properties. However, in its current version, the framework outputs a collection of dynamic strategies with fixed parameters and constant weights defining capital allocations. This excludes any adaptive regime adjustment or switching on the portfolio level for additional profitability from regime-specific patterns. In this work we extend boosting-based optimization framework by including capability to discover portfolio strategies with continuous and adiabatically smooth adjustment to the current market microregime. Such regime adaptivity is naturally provided by the input-dependent boosting. The proposed generalization preserves clarity and interpretability of the original framework since the dynamic base strategies of the multi-component portfolio and their optimal parameters remain fixed. However, the weights of the base strategies are adaptively varied in time according to the implicit rule discovered by boosting. Operational details of the new framework and encouraging results are illustrated using real market data. More rigorous theoretical foundation for the general concept of the boosting-based optimization is also outlined.

Keywords: adaptive boosting, ensemble learning, regime switching, trading strategies, portfolio optimisation.
\end{abstract}




\section{Introduction}

One of the most pronounced challenges in financial markets modeling and forecasting is nonstationarity of the individual time series and co-dependency relations. Usually it is very difficult or practically impossible to find a single global model based on observable and well-defined variables with desired performance in all market regimes. However, with certain degree of simplification such complex nonstationary dynamics can be approximated as switching between different regimes of market dynamics where each such regime is relatively simple and easy to describe by its own parsimonious model. More generally, one can introduce additional non-observable (hidden) state variables to represent market regimes and dynamical transitions between them.

Intuitively, timely switching or smooth transition between different regimes in such structured models could significantly improve modeling and forecasting of the nonstationary time series. More importantly, this may help to create dynamic trading strategies that consistently exploit regime-specific market patterns. However, although introduction of the hidden variables in the simplified econometric models [1] could lead to better prediction accuracy and overall explanatory power, the usefulness of such models for the discovery of the realistic trading strategies is often very limited. Moreover, potential of model improvement through the introduction of the finer structure of the market regimes (states) is also very limited since adequate model estimation is possible only when historical training data contains enough transitions between different regimes which is often not the case in most financial applications.

Certain simplifications, a priory assumptions and other drawbacks of the typical econometric models can be alleviated using dynamic machine learning approaches. For example, recurrent neural networks (NN) maintain an adaptive internal memory of past inputs that allows implicit regime adjustment or switching and do not require any specific assumptions about hidden state variables and mechanisms of regime transition [2-4]. However, training procedures for such NNs are often significantly more complex and less stable compared to already standard feed-forward NNs such as multi-layer perceptron (MLP) that are not dynamic [2,4]. Moreover, complex dynamic NNs lack interpretability and operational stability control that are very important requirements in financial applications.

Both statistical and machine learning forecasting models are trained using objectives that are not directly relevant to the trading strategy which limits usefulness of such models even when formal forecasting performance measures are reasonable [5]. Technical trading strategies, directly optimized to achieve desirable profit/loss (PL) distributions, usually have more practical value than pure forecasting models mentioned above. However, single trading strategy with reasonable complexity (to ensure out-of-sample performance) still cannot warranty stability across different market regimes.

Many modern automatic systems for systematic trading offer an option for the dynamic capital reallocation among different strategies using explicit userspecified rule that may be a function of the most recent PL time series generated 
by each strategy and other factors. The goal of such approach is to alleviate limitations of the single trading strategy that may be profitable only in certain market regimes while maintaining simplicity and interpretability of such portfolio of trading strategies. However, these systems do not provide any generic and theoretically-sound frameworks capable to discover such regimeadjusted portfolio strategies with stable performance over wide range of market regimes. For example, usage of resonance strategies tuned to the specific market regimes and simplified empirical switching between different strategies could be very unstable since it requires a very accurate timing which is difficult to achieve in most modern markets.

Recently proposed boosting-based optimization provides a generic framework for the discovery of portfolios of trading strategies with stable (non-resonant) performance over wide range of market regimes using intelligent combination of the complimentary, low-complexity base strategies with well-known properties [6-8]. This framework offers practical solutions for many problems encountered in other approaches. However, in its current version, the framework outputs a collection of dynamic strategies with fixed parameters and constant weights defining capital allocations. This excludes any adaptive regime adjustment or switching on the portfolio level for additional profitability from regime-specific patterns. In this work we extend boosting-based optimization framework by including capability to discover portfolio strategies with continuous and adiabatically smooth adjustment to the current market micro-regime. In many practical settings, the proposed framework could resolve or alleviate limitations of other approaches used for discovery of the regime-adjusted portfolio strategies.

\section{Limitations of the existing regime-switching and regime-adjusted models}

A flexible and generic approach to incorporate multiple interchanging regimes or states is to use models with hidden variables, i.e., auxiliary variables that are not directly observable [9]. A well-known example of such a framework is hidden Markov models (HMM) that are successfully used alone or in combination with other statistical or machine learning algorithms in speech recognition systems [10], bioinformatics [11], and other applications. Examples of hidden variable models used in financial econometrics include regime-switching models, the GARCH family of models, and credit risk models, where hidden variables are used to represent different economic/market regimes, volatility, and credit worthiness, respectively [1].

One of the widely used types of models with hidden variables is a linear statespace model that can be written in the following way [1]:

$$
\begin{aligned}
& y_{\tau}=A z_{\tau}+B x_{\tau}+\varepsilon_{\tau} \\
& z_{\tau+1}=C z_{\tau}+D x_{\tau}+\eta_{\tau}
\end{aligned}
$$


Here $x_{\tau}, y_{\tau}$, and $z_{\tau}$ are the vectors of deterministic inputs, observable outputs, and latent (nonobservable) state variables, respectively. Observation and transition equation white noise are given by $\varepsilon_{\tau}$ and $\eta_{\tau}$. Observation matrix, input matrix of the observation equation, transition matrix, and input matrix to the transition equation are given by $A, B, C$, and $D$.

A typical maximum likelihood (ML) based estimation for (1) would require approximate calculation of the nonobservable states $z$, which enters corresponding likelihood expression together with observable variables. Kalman filter [12] is usually used to provide optimal estimation of the state variables. Originally, Kalman filter was designed as an adaptive filter that provide optimal estimation and forecasting of the "true" states of the dynamical system from the multi-dimensional noisy observations in engineering applications. The filter is initialized with the initial conditions and computations are carried out recursively to the desired time, i.e. full historical information is used to make current estimation and forecasting.

The intuitive notion of the "states of the market" (e.g. bull, bear, and side markets) is well known to market practitioners. From a modeling perspective, this implies that different models and strategies should be used in different market states. If we add rules that prescribe the switching from one model (or set of models) to another one when market state changes, we arrive at the regimeswitching model $[1,13]$.

A broad class of regime-switching models are the Markov-switching vector autoregressive models (MS-VAR) [1,14]. Markov-switching model is a VAR model whose coefficients are driven by a Markov chain:

$$
y_{\tau}=\mu(s)+\left(\sum_{i=1}^{p} A_{i}(s) L^{i}\right) y_{\tau}+\varepsilon_{\tau}(s),
$$

where the matrices $A_{i}(s)$ are the coefficients of the process at lag $i$ in state $s$ and the noise terms $\varepsilon_{\tau}(s)$ are independent normal variables. The process is driven by a $k$-states Markov chain. A Markov chain is a discrete variable which can assume at each instant one of $k$ possible values with transition probabilities:

$$
P\left(s_{\tau}=i \mid s_{\tau-1}=j\right)=p_{i j}
$$

The realized state $s$ determines the coefficients and the vector of the intercepts of the process at each moment, so that the innovation term of the process is distributed as a mixture of Gaussian distributions. The state variable that could be used to represent market regime is a hidden factor. Due to the state (regime) switching MS-VAR becomes a nonlinear model even though it is based on linear components.

ML-based estimation of the MS-VAR model is presently the mostly widely used approach [1]. Similar to space-state model (1) and other models with hidden variables, one writes a likelihood function that depends on both observables and hidden variables. In the case of linear state-space models (1), Kalman filter is naturally applicable and used to estimate values of hidden variables (states). However, in nonlinear MS-VAR models, Expectation-Maximization (EM) algorithm is used instead. The algorithm is an optimization procedure for finding 
the maximum of log-likelihood functions in the presence of missing or hidden variables [15]. The idea of EM algorithm is to iterate between the estimate of the best parameters given the missing (hidden) data and the estimate of the missing (hidden) data given the best estimate of parameters.

The obvious limitation of econometric models with hidden state variables mentioned above is the usage of the simplified and pre-specified functional forms as in (1) and (2), (3). Compared to similar econometric models without hidden variables, the requirements for the data sample size will grow much faster with the model complexity increase. For example, even for simple regimeswitching model given by (2)-(3), the increase of the regime number beyond 2 or 3 could make adequate estimation of the model unrealistic since the available training data would not have enough transitions between different regimes to ensure reasonable out-of-sample performance. Moreover, even when formal forecasting performance measures are reasonable for explanatory purposes or large-scale risk management applications, the direct usefulness of the econometric-type models to trading strategies may often be limited due to the difference in objectives [5].

Machine learning approaches offer significantly more flexibility in many different applications including modeling of the non-stationary or multi-regime time series. However, the well-known feed-forward NNs such as widely used MLP with standard back-propagation training algorithm may be used to perform nonlinear prediction only on stationary time series. It is done by using delayed vector of inputs as follows:

$$
y_{\tau}=F\left(x_{\tau-1}, x_{\tau-2}, \ldots, x_{\tau-p}\right)
$$

Here $F$ is a general functional dependence that $\mathrm{NN}$ will try to approximate when $p$ delayed input variables will be presented at the input and one output variable will be generated at the $\mathrm{NN}$ output. The adequate choice of the lag space could be a difficult problem-dependent task. However, after the lag space is chosen, it remains constant. This limits NN's adaptivity and makes it a static model which can describe only stationary time series.

To make NN dynamic it must be given an "internal" memory [16]. To accomplish this and to retain simplicity of the feed-forward architecture, one can introduce time delays into the synaptic structure of the $\mathrm{NN}$ and to adjust their values during the learning phase. Such time delays are also neurobiologically motivated. One of such practical models where MLP with synapses represented by a finite-duration impulse response (FIR) filter was introduced by Wan [3]. The efficient training procedure called temporal back-propagation was also proposed for such FIR MLP [2,3]. One of the examples when effectiveness of the FIR MLP was demonstrated is the winning of the time series prediction competition [17].

A more general dynamic model denoted as a recurrent $\mathrm{NN}$ is obtained when the connectivity of the feed-forward network is extended to include feedback connections from the outputs of the units back to their inputs $[2,4]$. The most general recurrent $\mathrm{NN}$ is obtained if the output from every unit in the $\mathrm{NN}$ is fed back to the inputs of all units. The advantage of the recurrent NNs compared to 
the feed-forward NNs is due to an internal memory of past inputs introduced by the feedback connections. This internal memory is adaptive, i.e., during training it may be adapted to encompass those previous inputs which are relevant to the current problem. Such adaptive memory may completely relieve the user from specifying a lag space, since a fully recurrent $\mathrm{NN}$ is able to work entirely from its own internal memory, created from only a single external input [4]:

$$
y_{\tau}=F\left(x_{\tau-1}\right)
$$

However, despite their advantages recurrent NNs have not gained popularity similar to that of feed-forward NNs. Generally, it is more difficult to handle recurrent NNs in practice. In particular it has been found that training using widely accepted algorithms (e.g., gradient descent method and its extensions) is not sufficiently "powerful" to train recurrent NNs [4]. The problem could be in slow convergence or in complete failure to provide practically acceptable solution.

Even when training challenges are resolved, the remaining common problems with NNs and similar black-box machine learning models are poor interpretability and occasional instable behavior which is hard to control and predict. Although these drawbacks are especially undesirable in financial applications, the high noise-to-signal ratio in financial time series enhances probability of unstable behavior of such models.

One of the machine learning approaches to compensate for deficiency of the individual models is to combine several models to form a committee $[18,19]$. Committee can compensate limitations of the individual models due to both incomplete data and specifics of the algorithms (e.g., multiple local minima in the NN error surface). A number of different ensemble learning techniques to build optimal committees have been proposed over the years in different research communities [e.g., 6 and references therein]. The most relevant framework of this type in the context of the regime-switching or regime-adjusted models is mixture of expert (ME) model [20].

The probabilistic form of ME model can be written as [18]

$$
p(y \mid x)=\sum_{t=1}^{T} \pi_{t}(x) p_{t}(y \mid x)
$$

Here mixing coefficients $\pi_{t}(x)$ are known as gating functions and the individual component densities $p_{t}(y \mid x)$ are called experts. This terminology is due to the fact that different components can model the distribution in different regions of input space (they are "experts" at making predictions in their own regions), and the gating functions determine which components are dominant in which region. If the experts are linear (regression or classification) models, then the whole model can be fitted efficiently using the EM algorithm. An even more flexible model is obtained by using a multilevel gating function to give the hierarchical mixture of experts (HME) model [18]. This model can be imagined as a mixture distribution in which each component in the mixture is itself a mixture distribution.

More complex machine learning models can be used as components in both ME and HME frameworks. For example, NNs can be used as experts as well as 
gating functions [2]. However, increasing complexity of the models could often lead to practical problems in training of such multi-component system and to poor out-of-sample performance and instability. Thus, it is highly desirable to have committee of the well-understood and low-complexity expert models that consistently demonstrate acceptable combined performance.

Adaptive boosting is a powerful ensemble learning algorithm that combines many desirable features [21,22,19]. Many ensemble learning algorithms including "random sample" techniques like bagging can reduce only variance part of the model error, i.e. they make a combined model more stable. Boosting, on the other hand, can reduce both bias and variance parts of the model error. It means that one can start with simple model ("rule of thumb") with low accuracy and produce committee with much higher accuracy. Therefore, boosting can be applied to the pool of the well-understood low-complexity models to produce qualitatively different but interpretable combined model with significantly higher accuracy and stability [6-8]. Moreover, boosting tries to maximize margin to ensure good out-of-sample performance, i.e. it is a large-margin classifier $[21,22,19]$.

In our previous works [6-8] we propose a boosting-based optimization framework for the discovery of the stable portfolios of trading strategies from the low-complexity base strategies. It was argued that boosting for classification can be used as a basis for such stage-wise optimization framework. The final output of the boosting-based optimization is a collection $S$ of complimentary base trading strategies $B S_{i}$ with optimal parameter vectors $p_{i}$ and combination weights $w_{i}$ that specify capital allocation for each strategy $[7,8]$ :

$$
S \rightarrow\left\{\left[w_{1}, B S_{1}\left(p_{1}\right)\right], \ldots,\left[w_{T}, B S_{T}\left(p_{T}\right)\right]\right\}
$$

We have shown empirically $[7,8]$ that boosting-based optimization can generate portfolios (7) with stable performance over wide range of market regimes while using just well-known technical base strategies. However, in its current version, the framework outputs a collection of dynamic strategies (7) with fixed parameters $\boldsymbol{p}$ and constant weights $\boldsymbol{w}$. This excludes any adaptive regime adjustment or switching on the portfolio level for additional profitability from regime-specific patterns. Many modern automatic systems for systematic trading offer an option for the dynamic capital reallocation among different strategies in (7) using explicit user-specified rule. However, these systems do not provide any generic and theoretically-sound frameworks capable to discover regime-adjusted portfolio strategies with stable performance over wide range of market regimes. Switching-rule specification based just on the semi-quantitative discretional arguments could often lead to the unreliable trading system.

In this work we extend boosting-based optimization framework by including capability to discover portfolio strategies (7) where weights $\boldsymbol{w}$ are continuously adjusted by the implicit rule discovered by the input-dependent boosting. In the next two sections we summarize our previous results on the boosting-based optimization as well as provide more formal theoretical foundation for the framework. After that we introduce extended framework based on the inputdependent boosting [23] and present regime-adjusted trading strategy obtained from the new framework using real-market data. 


\section{Boosting-based optimization: discovery of the regime-independent portfolio strategies}

As described in our previous works [6-8], boosting for optimization could be based on different boosting frameworks. However, the generalized AdaBoost algorithm for classification [21,22] could be a reasonable choice in many applications due to its simplicity, comprehensive theoretical foundation, and proven robust performance in a large number of realistic classification problems.

For our purposes it is sufficient to describe boosting algorithm only for twoclass classification problem, where classifier outputs either +1 or -1 . Generalized AdaBoost for two-class classification consists of the following steps [22]:

$$
\begin{gathered}
w_{n}^{1}=1 / N \\
\varepsilon_{t}=\sum_{n=1}^{N}\left(w_{n}^{t} I\left(-y_{n} h_{t}\left(x_{n}\right)\right)\right) \\
\gamma_{t}=\sum_{n=1}^{N}\left(w_{n}^{t} y_{n} h_{t}\left(x_{n}\right)\right) \\
\alpha_{t}=\frac{1}{2} \ln \left(\frac{1+\gamma_{t}}{1-\gamma_{t}}\right)-\frac{1}{2} \ln \left(\frac{1+\rho}{1-\rho}\right) \\
w_{n}^{t+1}=w_{n}^{t} \exp \left(-\alpha_{t} y_{n} h_{t}\left(x_{n}\right)\right) / Z_{t} \\
H(x)=\sum_{t=1}^{T} \alpha_{t} h_{t}(x) / \sum_{t=1}^{T} \alpha_{t}
\end{gathered}
$$

Here $N$ is a number of training data points, $x_{n}$ is a model/classifier input set of the $n$-th data point and $y_{n}$ is the corresponding class label (i.e., -1 or +1$), I(z)=0$ for $z<0$ and $I(z)=1$ otherwise, $T$ is a number of boosting iterations, $w_{n}{ }^{t}$ is a weight of the $n$-th data point at $t$-th iteration, $Z_{t}$ is weight normalization constant at $t$-th iteration, $h_{t}\left(x_{n}\right)->[-1 ;+1]$ is the best base hypothesis/model at $t$-th iteration, $\rho$ is a margin control parameter, and $H(x)$ is a final weighted linear combination of the base hypotheses.

Boosting starts with equal and normalized weights for all training data (step (8.1)). A base classifier $h_{t}(x)$ is trained using weighted error function $\varepsilon_{t}$ (step (8.2)). If a pool of several types of base classifiers is used, then each of them is trained and the best one (according to error function) is chosen at the current iteration. The training data weights for the next iteration are computed in steps (8.3)-(8.5).

According to (8.5), at each boosting iteration, data points misclassified by the current best model (i.e., $y_{n} h_{t}\left(x_{n}\right)<0$ ) are penalized by the weight increase for the next iteration. In subsequent iterations, AdaBoost constructs progressively more difficult learning problems that are focused on hard-to-classify patterns. This process is controlled by the weighted error function (8.2). 
Steps (8.2)-(8.5) are repeated at each iteration until stop criteria $\gamma_{t}<\rho$ (i.e., $\varepsilon_{t}>=1 / 2(1-\rho)$ ) or $\gamma_{t}=1$ (i.e., $\varepsilon_{t}=0$ ) occurs. Step (8.6) represents the final combined (boosted) model that is ready to use. The model classifies unknown sample as class +1 when $H(x)>0$ and as -1 otherwise.

Portfolio strategy discovery is a direct optimization rather than classification problem. However, it was argued [6-8] that for a large class of objective functions, boosting for classification (8.1) - (8.6) can be efficiently used as a basis for the framework that could be labeled as "boosting for optimization" or "boosting-based optimization".

One of the natural and robust objectives for the trading strategy optimization is to require returns $(r)$ generated by the strategy on a chosen time horizon $(\tau)$ to be above certain conservative threshold $\left(r_{c}\right)$. By calculating strategy returns on a series of intervals of length $\tau$ shifted with a step $\Delta \tau$ and encoding them as +1 (for $r>=r_{c}$ ) and -1 (for $r<r_{c}$ ), one obtains symbolically encoded distribution of strategy returns.

Contrary to the classification problems, here the purpose is not to minimize classification error, but rather to minimize the number of -1 samples, i.e., number of subcritical returns. This can still be considered as classification problem with potentially uneven sample number between two classes. The described objective can be incorporated into the boosting operation $(8.1)-(8.6)$ by considering output -1 as misclassification. In such setting, boosting (8.1) - (8.6) provides a framework for optimization, where minimization objection function is a "miss rate", i.e., number of -1 samples divided by the total number of samples. The objective function can be generalized to include any complex condition with combination of different objectives for profit maximization and risk minimization.

In the case of trading strategy optimization, the final usage of boosting output is different from the classical case of boosting for classification. Instead of using weighted linear combination (8.6) of the base models as a final model for classification, one uses boosting weights to construct portfolio of strategies. The initial capital is distributed among different base strategies in amounts according to the weights $\left(\alpha_{t} / \Sigma \alpha_{t}\right)$ obtained from boosting which are already normalized.

As discussed in [6-8], boosting can be used to discover the optimal combination of different dynamic trading strategies for a single financial instrument as well as the simultaneous combination of trading strategies and different instruments. In both cases, boosting steps $(8.1)-(8.6)$ are applied to a pool of base strategies $\left\{B S_{i}\left(\boldsymbol{p}_{i}\right)\right\}$, where $\boldsymbol{p}_{i}$ is a vector of adjustable parameters for strategy $B S_{i}$. However, in the first case all base strategies are applied to a time series of a single financial instrument $F I_{0}$, while set $\left\{B S_{i}\right\} \times\left\{F I_{j}\right\}$ of all possible pairs of strategies $B S_{i}$ and instruments $F I_{j}$ should be used in the latter case.

According to error function (8.2), if the objective is to maximize the number of supercritical returns on the shifted intervals, the following optimization problems are solved for all base strategies $B S_{i}\left(\boldsymbol{p}_{i}\right)$ and financial instruments $F I_{j}$ at each boosting iteration:

$$
\min _{p_{i}}\left[\sum_{n=1}^{N} w_{n}^{(t)} I\left(r_{c}-r_{n}^{\tau}\left(B S_{i}\left(p_{i}\right), F I_{j}\right)\right)\right]
$$


Here, $r_{n}{ }^{\tau}$ is a return produced by the strategy $B S_{i}\left(p_{i}\right)$ applied to the instrument $F I_{j}$ over $n$-th shifted interval of length $\tau$ and $r_{c}$ is a chosen threshold value. Often linear function $\sim\left(r_{c}-r_{n}\right)$ is better choice for $z<0$ compared to standard stepfunction $I$. Based on the results of these minimization procedures for all $(i, j)$ pairs, the best pair "strategy-instrument" of the current iteration is added to the portfolio. This procedure was also generalized to allow simultaneous discovery of new synthetic instruments expressed as multiple spreads between base instruments and dynamic trading strategies for such spreads [8].

The AdaBoost algorithm given by (8.1) - (8.6) is one of the classical versions and it was described as an example suitable for boosting-based optimization in all our previous works. However, a slightly different version is both more natural and more convenient for the extension to the input-dependent boosting [23]. For simplicity, we will omit the margin parameter $\rho$ in all the following formulations. The other standard AdaBoost formulation given in [23], results in a different weight update procedure. Instead of (8.5) one applies:

$$
w_{n}^{T}=\frac{e^{-H_{T-1}\left(x_{n}\right) y_{n}}}{\sum_{j=1}^{N} e^{-H_{T-1}\left(x_{j}\right) y_{j}}}
$$

Expression for $\alpha$ is given by the following form equivalent to (8.4) with omitted margin parameter:

$$
\alpha_{T}=\frac{1}{2} \ln \left(\frac{\sum_{n=1}^{N} w_{n}^{T} I\left(h_{T}\left(x_{n}\right), y_{n}\right)}{\sum_{n=1}^{N} w_{n}^{T} I\left(-h_{T}\left(x_{n}\right), y_{n}\right)}\right)=\frac{1}{2} \ln \left(\frac{1-\varepsilon_{T}}{\varepsilon_{T}}\right)
$$

As mentioned in our previous publications [6-8], boosting also allows natural and easy incorporation of other ensemble learning techniques on the inside and outside levels for further improvement of the accuracy and stability. For example, at each boosting iteration, instead of choosing a single best model, one can choose mini-ensemble of models using other ensemble learning techniques. In the simplest case, this could be an equal weight mini-ensemble of several comparable best models. One can also use boosting in the HME-like framework by building portfolio of portfolios.

As summarized in this section, it is quite intuitive to use boosting for classification as a basis for the boosting-based optimization framework. However, to better understand basic features of the boosting-based optimization and its possible generalizations and limitations, it is still useful to outline formal theoretical foundation for the framework. A short version of such foundation is presented in the next section.

\section{Theoretical foundation for boosting-based optimization}

Portfolio strategy discovery is a direct optimization rather than classification problem. However, several formal arguments presented in this section should clearly demonstrate that boosting for classification can be naturally generalized 
for the framework of boosting-based optimization as was suggested in our previous papers [6-8].

One of the operational interpretations of the boosting algorithm shows that boosting fits an additive logistic regression model by a stage-wise optimization of expected exponential loss [24]:

$$
\mathrm{E} e^{-y F(x)} \rightarrow \min
$$

One of the motivations for such choice of the loss function is that exponential loss is an upper bound on the classification error [25]:

$$
P[y F(x)<0]<\mathrm{E}^{-y F(x)}
$$

This kind of upper bound can be generalized for any event $A$ :

$$
P[A] \leq \mathrm{E} e^{-\left(2 I_{A}-1\right)|F(x)|},
$$

where $I_{A}=1$ if $A$ takes place and $I_{A}=0$ otherwise. In the case of standard classification problem event $A$ describes a misclassification, i.e. classification error.

In the case of multi-component portfolio strategy optimization let us consider an event "return of a combined strategy $S$ is lower than a threshold $r_{c}$ ", i.e. $r(S(x))<r_{c}$. Then inequality (14) can be written as

$$
P\left[r(S(x))<r_{c}\right] \leq \mathrm{E} e^{-\left(2 I_{\left[r(S(x))<r_{c}\right]}-1\right)\left|r(S(x))-r_{c}\right|}
$$

Looking at the right part of inequality (15) we can see that optimization task can be reduced to classification task in a way proposed in [6-8]. Thus, we get a generalization of boosting framework for optimization tasks, where instead of minimizing classification error we are trying to minimize a number of intervals where $\left[r(S(x))<r_{c}\right]$. Minimization of $P\left[r(S(x))<r_{c}\right]$ is equivalent to the maximization of $P\left[r(S(x))>=r_{c}\right]$.

Boosting is known to demonstrate stable generalization abilities measured by its performance on out-of-sample data. Standard Vapnik-Chernovenkis (VC) analysis [26] of boosting bounds generalization loss by empirical risk (here $\hat{\operatorname{Pr}}[\bullet]$ denotes empirical probability measured on the training set) and a term dependent on VC-dimension of weak learner and number of boosting iterations [27]:

$$
P[H(x) \neq y] \leq \hat{\operatorname{Pr}}[H(x) \neq y]+\tilde{O}\left(\sqrt{\frac{T d}{m}}\right)
$$

where $d$ is the VC-dimension (capacity) of weak learner, $m$ is the size of the training set, and $T$ is the number of weak classifiers in the committee. However, this bound could often contradict with practical experience, which shows that boosting can decrease its test error even when empirical risk is already zero.

The best explanation of boosting generalization capabilities so far is margin theory [28]. Margin theory provides an upper generalization bound independent of number of iterations made by boosting. This bound suggests that boosting may not overfit even if ran for many rounds. For any $\theta$ : 


$$
P[H(x) \neq y] \leq \hat{\operatorname{Pr}}[\operatorname{margin}(x, y) \leq \theta]+\tilde{O}\left(\sqrt{\frac{d}{m \theta^{2}}}\right),
$$

where margin is

$$
\operatorname{margin}(x, y)=\frac{y \sum_{t} \alpha_{t} h_{t}(x)}{\sum_{t} \alpha_{t}} .
$$

Margin is the measure of confidence of a boosted predictor in its decision. It is positive if the decision is correct and negative otherwise. Theory states that for better generalization one should increase the margins of training samples.

In the context of the trading strategy boosting margin can be reformulated as

$$
\operatorname{margin}\left(r_{n}^{\tau}\right)=\frac{\sum_{t} \alpha_{t} I\left(r_{c}-r_{n}^{\tau}\left(B S_{t}\left(p_{t}\right), F I_{t}\right)\right)}{\sum_{t} \alpha_{t}}
$$

In such interpretation we want to maximize the confidence of our boosted strategy for over each of $n$ shifted intervals of length $\tau$. In order to strictly apply theory for strategy boosting, some additional statements should be proven (e.g., finite capacity of base strategies - analogous to VC-dimension). Although the rigorous proof is out of scope of this paper, the usage of the low-complexity base strategies should not violate these assumptions in most practical settings.

Under some conditions on a weak learner, it was proven that boosting is a regularized search for a maximum margin solution [29]. That means that in the limit, the boosted committee may have a maximum possible lower margin. Recent studies of boosting suggest that one may expect overfitting in case of increasing VC-dimension of weak learner through iterations [30], or in the case of the overlapping class distributions [31]. However, usage of the lowcomplexity base strategies should be able to alleviate these problems in most practical applications.

\section{Optimization framework based on input-dependent boosting: Discovery of the regime-adjusted portfolio strategies}

Majority of the known boosting algorithms including classical AdaBoost assume that the combination weights $\alpha_{t}$ are fixed constants and therefore do not take particular input patterns into consideration. Application of such algorithms as a basis for the boosting-based optimization allows discovering portfolio strategies with fixed weights, i.e., fixed capital allocations among the individual strategies. This excludes any dynamic regime-adjustment to exploit regime-specific patterns for further increase of the portfolio strategy profitability.

However, several input-dependent boosting algorithms have been recently proposed [23,32,33]. One of these algorithms (called WeightBoost) [23] seems to be the most appealing practical choice due to its close relation to the original AdaBoost and flexibility to vary the form and degree of the regime adjustment. 
WeightBoost not only introduces input dependency but also provides practical regularization mechanism that helps to avoid overfitting problems that boosting may encounter in applications with high level of noise.

The original motivation of this input-dependent boosting algorithm [23] is to alleviate two limitations of the classical AdaBoost and its extensions. One is constant combination weights that prevent from using full potential of the base models in the regimes of their expertise. The other problem is potential overfitting in high-noise cases that requires introduction of the practical and efficient regularization. However, most of the previously proposed regularization algorithms are unsuccessful or only partially successful either due to the high computational cost or lack of strong empirical results of improvement.

The following inductive form of the boosted classifier is used in AdaBoost derivation and its input-dependent extension given in [23]

$$
H(x)=H_{T-1}(x)+\alpha_{T} h_{T}(x)
$$

The potential overfitting problem of AdaBoost can be implied from the weight updating function (10). If there are some noisy data patterns that are difficult to classify correctly by the base classifier(s), the value of $-H_{T-1}\left(x_{i}\right) y_{\mathrm{i}}$ for those data points will accumulate linearly (since $H(x)$ is a linear combination of base classifiers) and the corresponding weights will grow exponentially. Therefore, the particular sampling procedure within the AdaBoost algorithm will overemphasize the noisy training data points and may lead to poor generalization.

Since the potential overfitting is caused by the accumulation of errors within the function $H_{T-1}(x)$, one way to avoid this is to modify the expression form for $H_{T-I}(x)$. Instead of constant combination coefficients $\alpha_{t}$, we can make them input dependent, i.e.

$$
H_{T}(x)=\sum_{t=1}^{T} \alpha_{t} e^{-\left|\beta H_{t-1}(x)\right|} h_{t}(x)
$$

Compared to (20), the above expression replaces the weighting constant $\alpha_{t}$ with $\alpha_{t} \exp \left(-\left|\beta H_{t-1}(x)\right|\right)$. More interestingly, it can be shown that when $\alpha_{t}$ is bounded by some fixed constant, the value of $H_{T}(x)$ in (21) will increase at most logarithmically with respect to the number of iterations $T$ and the weight of each data pattern will grow at most polynomial with the number of iterations. Therefore, the problem of overemphasizing noisy data patterns will be alleviated substantially compared to the classical AdaBoost.

As pointed out before the other important problem with AdaBoost is fixed combination coefficients. According to AdaBoost, each base classifier (model) $h_{t}(x)$ is trained intentionally on the data patterns that either misclassified or weakly classified by previous classifiers (models) $H_{t-1}(x)$. Therefore, every base classifier $h_{t}(x)$ should be appropriate only for a subset of input patterns. However, in the prediction phase, the opinion of the base classifier $h_{t}(x)$ will always be weighted by the same number $\alpha_{t}$ no matter what test examples are. 
On the contrary, in the new form of $H_{T}(x)$ given by (21), introduction of the instance-dependent factor $\exp \left(-\left|\beta H_{t-1}(x)\right|\right)$ offers a tradeoff between the opinion of the base classifier $h_{t}(x)$ and that of the previously built meta-classifier $H_{t-1}(x)$. Since the value of $H_{t-1}(x)$ indicates its confidence on classifying the instance $x$, the factor $\exp \left(-\left|\beta H_{t-1}(x)\right|\right)$ forces to consider the opinion of $h_{t}(x)$ seriously only when combination of the previous classifiers $H_{t-1}(x)$ is not confident about its decision. This implies that introduction of the input-dependent factor makes the base classifier $h_{t}(x)$ to be consistent between the training phase and the prediction phase, i.e., $h_{t}(x)$ is used for prediction of the particular type of input patterns that it has been trained on.

It is clear from (21) that the extent of the input-dependency can be conveniently controlled by factor $\beta$. When $\beta$ goes to zero, we recover original AdaBoost algorithm. By varying $\beta$ one can go from small weight modulations (mild regime adjustment) in almost stationary meta-model (small $\beta$ ) to the more regime-switching type of meta-model (large $\beta$ ). The optimal choice of $\beta$ is determined by the properties that user expects from the meta-model and stability of its out-of-sample performance.

Finally, one needs to obtain a learning procedure that is able to minimize the exponential cost function with the new combination form given by (21). As shown by Jin et al [23], by following standard procedures for AdaBoost derivation, one obtains the new expression for the data weight modification at each boosting iteration:

$$
w_{n}^{T}=\frac{e^{-H_{T-1}\left(x_{n}\right) y_{n}-\left|\beta H_{T-1}\left(x_{n}\right)\right|}}{\sum_{j=1}^{N} e^{-H_{T-1}\left(x_{j}\right) y_{j}-\left|\beta H_{T-1}\left(x_{j}\right)\right|}}
$$

All other relevant expressions given by (8.1) - (8.5) remain the same, while the new combination form is given by $(21)$. Using similar arguments one can obtain learning procedure for an arbitrary bounded regularizer $f(x)$ instead of $\exp \left(-\left|\beta H_{t-1}(x)\right|\right)$ used above [23].

The described input-dependent WeightBoost algorithm consistently outperforms classical AdaBoost algorithm in many typical classification tasks [23]. However to adapt this input-dependent boosting for classification to the boosting-based optimization framework capable to discover regime-adjusted portfolio strategies, additional assumptions should be made. In the standard classification task one can run each base model for a given test data sample to compute each input-dependent combination weight according to (21). After that the output of the combined model (21) can be readily obtained. However, we do not know the base strategies performance (i.e., return) on the tested interval $\tau$ in advance and cannot compute input-dependent weights (capital allocations) before running the portfolio of strategies. Therefore, one should use information on the base strategies performance from previous intervals.

A natural way is to use return information on the most recent interval $\tau^{*}$ which could be different from $\tau$ used in the objective function (9) in the training phase. The interval $\tau^{*}$ is another parameter that can be varied to achieve specific 
objectives. For example, for aggressive local regime-switching strategies one can use short period $\tau^{*}$, while for a slow (adiabatic) regime-adjustment of the already stable portfolio one can use larger $\tau^{*}$. The final choice of $\tau^{*}$ is empirically determined by the strategy performance.

For some portfolio strategies, time variation of the capital allocations among individual strategies could introduce additional operational challenges. For example, for daily or other low-frequency trading strategies one would need to rebalance existing positions even when individual strategies do not generate new entry or exit signals since weight distribution changes according to latest performance of the base strategies. However, all these technical issues can be resolved for each type of portfolio strategy.

Intraday strategies with no overnight positions introduce the least number of operational challenges in the application of the regime-adjusted portfolio strategies obtained from input-dependent boosting. Indeed, if there are no intraday weight adjustments, all new capital allocations for the next day are computed using already existed performance data from the current day and chosen number of previous days. The example of such strategy is considered in the next section as an illustration of the new framework application to the realmarket problem.

\section{Application example}

Intraday trading strategies with no overnight positions is very important type of strategies in modern markets where many other strategies operating on lower frequency (i.e. daily) could often fail on a short-term horizon (e.g. several months). In addition, operational challenges imposed by the input-dependent boosting framework are minimal in this case. Here we use one of such strategies to illustrate application of the new framework.

The detailed empirical comparison of the previously studied boosting-based optimization with constant weights and new framework with adjustable weights is beyond the scope of this paper. Instead, using real example we summarize the features of the new framework that have been already found very useful in practical market applications. The presented results are mostly relevant to the strategies with slow (adiabatic) and limited-amplitude adjustments of the weights (capital allocations) which are the main focus of our current research. More aggressive, regime-switching strategies that can be profitable even in the cases where stable portfolio strategy with constant capital allocations cannot be found will be discussed elsewhere.

We apply boosting-based optimization to the single financial instrument and the pool of the typical momentum (trend-following) strategies operating on $5 \mathrm{~min}$ intraday bars. Each base strategy is based on the two types of entry indicators and two types of trailing stop exits that combine risk management and profittaking capabilities. Since each base strategy is restricted to only one side of entry (i.e., long-only or short-only) and number of entry trades allowed during the trade day is varied from 1 to 3 , the pool consists of 24 individual base strategies where parameters of the entry and exit rules are available for optimization. 
The entry indicators considered in this application are double exponential cross-up and relative strength index (RSI) [34]. Double exponential indicator consists of two exponential moving averages (EMA) of the $5 \mathrm{~min}$ bars with 2 parameters each (smoothing constant and averaging period). When price crosses up both EMAs, entry signal for long position is generated (cross-down signals short position). RSI entry rule have two parameters: period and threshold. When price crosses up the RSI threshold the long entry signal is generated (cross-down signals for short position).

Long (short) position is closed when $5 \mathrm{~min}$ bar closing price fells below $c_{\max }[1-\Delta \sigma(m)]$ (jumps above $\left.c_{\min }[1+\Delta \sigma(m)]\right)$. Here $c_{\max }\left(c_{\min }\right)$ is a maximum (minimum) closing prices with respect to present bar, $\sigma(m)$ is volatility measure computed using $m$ last bars, and parameter $\Delta$ controls trailing stop width. Parkinson volatility measure [35] was used in our case. Trailing stops based on global and local (i.e., the most recent) maximum (minimum) calculations used for the exit price level can demonstrate complimentary performance. In this example, both exit types have been used with parameters $\Delta$ and $m$ available for optimization.

In the presented example we considered 7 years (1750 days) of historical intraday data for the Russell-2000 (ticker: RUT) small-cap index ending on $2008 / 02 / 01$. Only 800 days ending on 2007/01/31 are used for training. All other data after (more than 1 year) and before $(\sim 3$ years) the training period is used to demonstrate out-of-sample stability of the discovered portfolio strategies. The search is restricted to the strategies operating on the last 30 bars of each trading day. Assuming realistic fees of $0.5 \mathrm{c}$ per share and stop loss slippage of $5 \mathrm{c}$ for IWM which is RUT-based tradable ETF with price ratio 1:10, we used $5 \mathrm{c}$ and 50c for RUT index itself. For clear and conservative demonstration of the daily profit-loss (PL) time series no reinvestment was used, i.e., each trading day was started with the same amount of trading capital. Both fixed-weight and inputdependent boosting optimization was restricted to total of 7 iterations. At each boosting iteration, base strategies were optimized using global simulated annealing optimizer [36].

The best portfolio strategies, discovered using fixed-weight boosting (8.1) (8.6) with the objective function (9) and different parameters $\tau$ and $r_{c}$, often demonstrate comparable global performance and the variability of the local performance is also quite limited. When we apply input-dependent boosting and vary parameter $\beta$ that controls regime-adjustment, the immediate result is increased diversity of the obtained portfolio strategies. The discovered regimeadjusted portfolio strategies may not always demonstrate overwhelming global superiority in comparison to the portfolio strategies obtained with fixed-weight boosting. However, as expected, they can demonstrate significantly superior performance in certain regimes and different mechanisms of profit generation.

For example, applying the fixed-weight boosting to the momentum strategies operating on broad market indexes, including Russell-2000, one often finds only portfolio strategies where almost all capital $(>90 \%)$ allocated to long-only base strategies. This may introduce occasional undesirable effects (e.g., daily drawdowns) in certain local regimes. However, we find that input-dependent 
boosting with even small values of $\beta$ is capable to discover much more diverse portfolio strategies including those where capital allocation to long and short strategies is either more uniform or oscillates between long and short dominating portfolios which improves performance in certain market regimes.

These results are illustrated in figure 1 where we plot PL time series of the typical portfolio strategy obtained using fixed-weight boosting (solid line), inputdependent boosting (dashed line), and benchmark buy-and-hold long strategy (dotted line). These solutions are obtained using the following objective function and boosting parameters: $\tau=15$ days, $r_{c}=0.2 \%$ (for fixed-weight boosting) and $\tau=\tau^{*}=15$ days, $r_{c}=0.1 \%, \beta=2$ (for input-dependent boosting). It is clear that although global performance of the two portfolio strategies is comparable, the regime-adjusted strategy demonstrates significant advantage in certain regimes. Both boosting strategies show very stable out-of-sample performance and are consistently superior to buy-and-hold strategy.

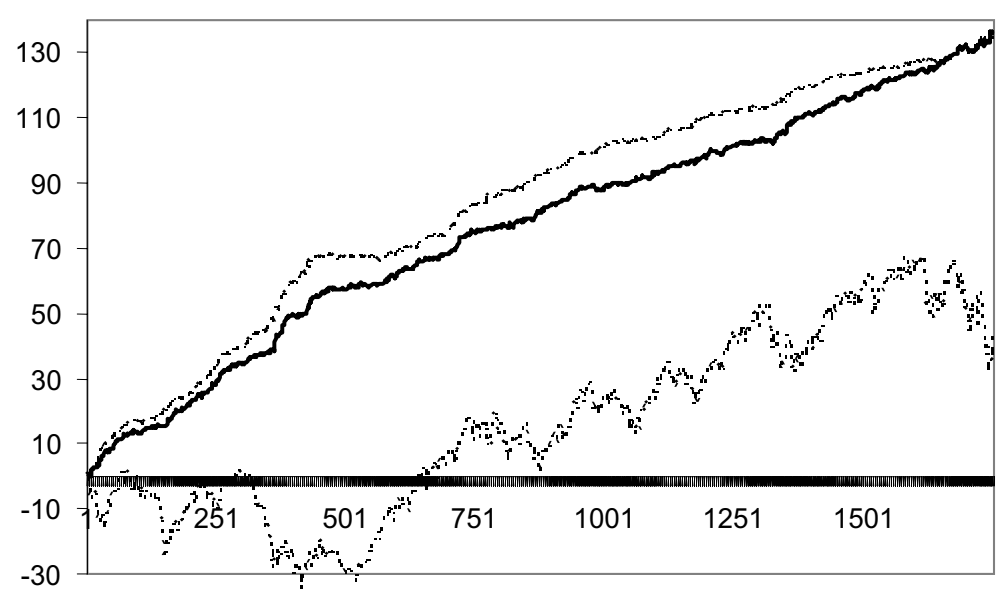

Figure 1: $\quad \operatorname{PL}(\%)$ vs days.

However, even more important is the ability of the input-dependent boosting to find much greater variety of the portfolio strategies without any additional efforts such as empirical search for more complex objective functions or any other non-generic and problem specific approaches. For example, long-short bias ratio of the regime-adjusted portfolio strategy presented in figure 1 varies from $\sim 0.75$ long bias to $\sim 0.7$ short bias in different regimes, while the fixed weight portfolio strategy is long-only at all times. The significant reduction of the correlation between these two portfolio strategies is clear from figure 2 where each point represent daily returns of the fixed-weight (horizontal axis) and adjusted-weight (vertical axis) boosted strategies. The ability to generate more diverse portfolio strategies would allow building more stable portfolios of portfolios using HME or similar frameworks. Additionally, using portfolio-level intraday profit-taking or stop losses, more specific return-risk profiles could be 
achieved. Initigl diversity of the boosted strategies will provide a practical advantage in this task. Finally, we found that input-dependent boosting is much more powerfud.5compared to fixed-weight boosting when one searches for some specific hard-to-discover strategies, e.g. short-only hedging strategy that minimally interferes with the original long strategy.

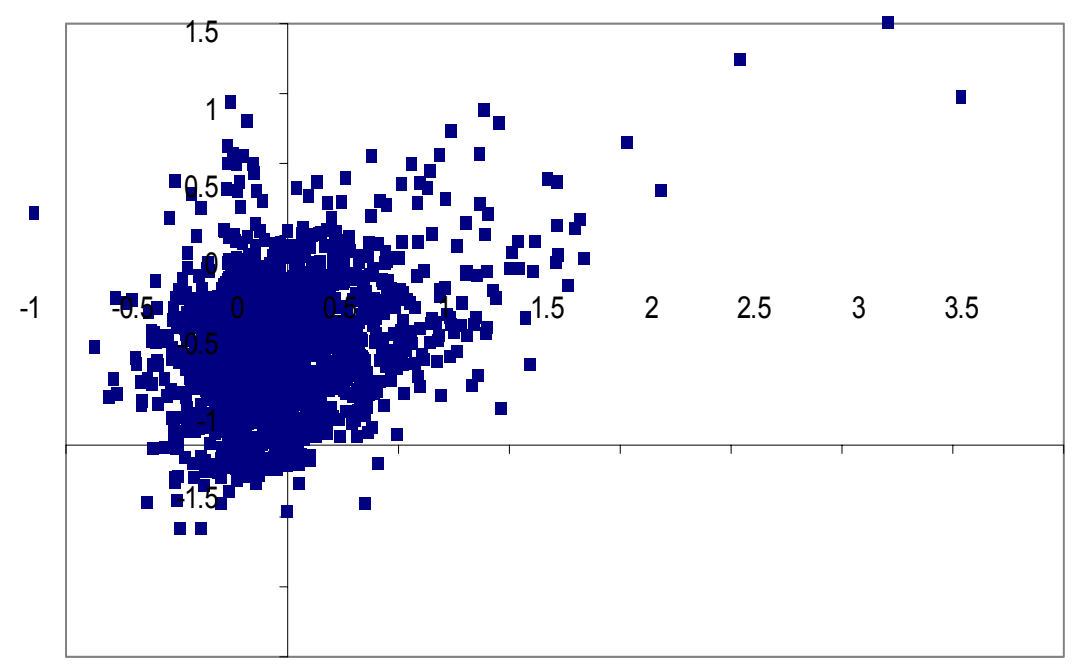

Figure 2: Daily returns(\%) (fixed vs adjusted weights).

\section{Conclusions}

Limitations of the existing regime-switching and regime-adjusted models and modelling frameworks for financial applications have been outlined. Recently proposed boosting-based optimization has been extended to allow discovering of the regime-switching and regime-adjusted portfolio strategies. A formal theoretical foundation for the boosting-based optimization in general has also been outlined. Operation of the new framework was illustrated using real-market example of the intraday trading strategy.

\section{References}

[1] Fabozzi, F.J., Financial modelling of the equity market: From CAPM to cointegration, Wiley, 2006.

[2] Haykin, S., Neural networks: A comprehensive foundation, Macmillian College Publishing Company, 1994.

[3] Wan, E.A., Finite impulse response neural networks with applications in time series prediction, PhD Dissertation, Stanford University, 1993.

[4] Pedersen, M.W., Optimization of the recurrent neural networks for time series modelling, PhD Thesis, Technical University of Denmark, 1997. 
[5] Gavrishchaka, V.V. \& Bykov, V., Market-neutral portfolio of trading strategies as universal indicator of market micro-regimes: From rare-event forecasting to single-example learning of emerging patterns, in proceedings of Second International Conference on Innovative Computing, Information and Control (ICICIC 2007).

[6] Gavrishchaka, V.V., Boosting-based frameworks in financial modeling: Application to symbolic volatility forecasting, Advances in Econometrics, 20B, p. 123, 2006.

[7] Gavrishchaka, V.V., Boosting-based framework for portfolio strategy discovery and optimization, New Mathematics and Natural Computation, 2, p. 315,2006

[8] Gavrishchaka, V.V., Discovery of multi-spread portfolio strategies for weakly-cointegrated instruments using boosting-based optimization, in JCIS-CIEF Proceedings, 2006.

[9] Cappe, O., Moulines, E., Ryden, T., Inference in hidden Markov models, Springer Series in Statistics, Springer, 2005.

[10] Rabiner, L.R., A tutorial on hidden Markov models and selected applications in speech recognition, Proc. IEEE, 77, 257-286, 1989.

[11] Baldi, P. \& Brunak, S., Bioinformatics: The machine learning approach, MIT Press, 2001.

[12] Kalman, R.E., A new approach for linear filtering and prediction problems, Transactions of the ASME-Journal of Basic Engineering 82, Series D, pp.35-45, 1960.

[13] Hamilton, J.D., A new approach to the economic analysis of the nonstationary time series and the business cycle, Econometrica, Econometric Society, 57(2), pp. 357-384, 1989.

[14] Zivot, E. \& Wang, J., Modeling financial time series with S-Plus, Springer, 2003.

[15] Dempster, A.P., Laird, N.M. \& Rubin, D.B., Maximum-likelihood from incomplete data via the EM algorithm, Journal Royal Statistical Society Series 39, pp. 1-38, 1977.

[16] Elman, J.L., Finding structure in time, Cognitive Science, 14, pp. 179-211, 1990.

[17] Wan, E.A., Time series prediction by using a connectionist network with internal delay lines, in Time series prediction: Forecasting the future and understanding the past (A.S. Weigend and N.A. Gershenfeld, eds.), pp. 195-217, Reading, MA: Addison-Wesley, 1994.

[18] Bishop, C.M., Pattern recognition and machine learning, Springer, 2006.

[19] Hastie, T., Tibshirani, R. \& Friedman, J., The Elements of Statistical Learning: Data Mining, Inference, and Prediction, Springer, 2001.

[20] Jacobs, R.A., Jordan, M.I., Nowlan, S.J. \& Hinton, G.E., Adaptive mixtures of local experts, Neural Computation, 3(1), pp. 79-87, 1991.

[21] Schapire, R.E., The design and analysis of efficient learning algorithms, PhD thesis, MIT Press, 1992.

[22] Ratsch, G., Robust boosting via convex optimization: Theory and applications, PhD thesis, Potsdam University, 2001. 
[23] Jin, R., Liu, Y., Si, L., Carbonell, J. \& Hauptmann A.G., A new boosting algorithm using input-dependent regularizer, Proc. of the $20^{\text {th }}$ International Conference on Machine Learning (ICML-2003), Washington DC, 2003.

[24] Friedman, J.H., Hastie, T. \& Tibshirani, R., Additive logistic regression: A statistical view of boosting, Annals of Statistics, 28, 2000.

[25] Schapire, R.E. \& Singer, Y., Improved boosting using confidence-rated predictions, Machine Learning, 37(3), 1999.

[26] Vapnik, V., Statistical learning theory, Wiley, 1998.

[27] Freund, Y., \& Schapire, R. E., A decision-theoretic generalization of online learning and an application to boosting, Journal of Computer and System Sciences, 55, pp. 119-139, 1997.

[28] Schapire, R. E., Freund, Y., Bartlett, P., \& Lee, W. S., Boosting the margin: A new explanation for the effectiveness of voting methods, The Annals of Statistics, 26, pp. 1651-1686, 1998.

[29] Rudin, C., Daubechies, I. \& Schapire, R.E., The Dynamics of AdaBoost: Cyclic Behavior and Convergence of Margins, Journal of Machine Learning Research, December 2004.

[30] Reyzin, L. \& Schapire, R.E., How boosting the margin can also boost classifier complexity, In Proceedings of the 23rd International Conference on Machine Learning, 2006.

[31] Vezhnevets, A. \& Barinova, O., Avoiding Boosting Overfitting by Removing 'Confusing Samples', European Conference on Machine Learning (ECML) - 2007, Warsaw, Poland, 2007.

[32] Altincay, H., A dempster-shafer theoretic framework for boosting based ensemble design, Pattern Anal. Applic., 8, pp. 287-302, 2005.

[33] Vetrov, D. \& Kropotov, D., Data dependent classifier fusion for construction of stable effective algorithms, Proc. of the $17^{\text {th }}$ International Conference on Pattern Recognition (ICPR-04), 2004.

[34] Katz, J.O. \& McCormick, D.L., The encyclopaedia of trading strategies, McGraw-Hill, 2000.

[35] Li, K. \& Weinbaum, D., The empirical performance of the alternative extreme value volatility estimators, Working Paper, Stern School of Business, New York, 2000.

[36] Press, W.H., Teukolsky, S.A., Vetterling, W.T. \& Flannery, B.P., Numerical Recipes in C: The Art of Scientific Computing, Cambridge University Press, 1992. 